\title{
Uma avaliação da satisfação de pacientes em hemodiálise crônica com o tratamento em serviços de diálise no Brasil
}

\author{
I ${ }^{1}$ Gisele Macedo da Silva, ${ }^{2}$ Isabel Cristina Gomes, \\ ${ }^{3}$ Elaine Leandro Machado, ${ }^{4}$ Fernando Henrique Rocha, ${ }^{5}$ Eli lola Gurgel Andrade, \\ ${ }^{6}$ Francisco de Assis Acurcio, ${ }^{7}$ Mariângela Leal Cherchiglia I
}

Resumo: O objetivo deste artigo foi avaliar a satisfação dos pacientes em tratamento hemodialítico crônico com o cuidado recebido nos serviços de diálise. Trata-se de estudo transversal desenvolvido a partir de amostra nacionalmente representativa de pacientes portadores de doença renal crônica terminal $(\mathrm{N}=3.036)$ em tratamento em serviços de diálise e centros transplantadores do Brasil. Os pacientes em hemodiálise (HD) responderam a um questionário de satisfação específico para essa modalidade, cujas questões envolveram satisfação com equipe, ambiente e cuidado prestado, e impacto da diálise nas atividades diárias. Para obter um escore de satisfação desses pacientes, foram utilizados modelos de Teoria de Resposta ao Item na análise dos 22 itens do questionário. A regressão linear foi utilizada para avaliar a associação entre fatores socioeconômicos, demográficos, clínicos e estruturais dos serviços de diálise e os escores de satisfação. Dos pacientes entrevistados, 1.621 estavam em HD no momento da entrevista. Todos responderam ao questionário de satisfação. A maioria era do sexo masculino, cor da pele não-branca, escolaridade $\leq 11$ anos e não relataram depressão. A idade média foi de 49 anos. A análise dos dados revelou que ter menor idade, ter depressão, possuir maior tempo de tratamento dialítico e despender maior tempo de deslocamento da residência até o serviço de diálise estiveram associados a menor nível de satisfação com os serviços de diálise. Sugere-se que, para avaliar a satisfação dos usuários, seja importante considerar, além dos fatores individuais, os atributos relacionados aos serviços de saúde, confirmando sua natureza multidimensional.
1 Odontóloga; especialista em Endodontia pela Pontifícia Universidade Católica de Minas Gerais; mestre em Saúde Pública pela Faculdade de Medicina da Universidade Federal de Minas Gerais (UFMG). Endereço eletrônico: gimacedosilva@hotmail.com

2 Estatística; mestre em Estatística pelo Instituto de Ciências Exatas da UFMG. Endereço eletrônico: bebel_ suty@yahoo.com.br

${ }^{3}$ Psicóloga; mestre em Saúde Pública pela Faculdade de Medicina da UFMG. Endereço eletrônico: elainemachado77@ yahoo.com.br

${ }^{4}$ Aluno de graduação da Faculdade de Medicina da UFMG. Endereço eletrônico: fernando_hro@hotmail.com

${ }^{5}$ Economista; doutora em Demografia pela UFMG: professora associada da Faculdade de Medicina da UFMG. Endereço eletrônico: iola@medicina.ufmg.br

${ }^{6}$ Médico; doutor em Ciência Animal pela UFMG; pós-doutor em Economia da Saúde e do Medicamento na Universitat Pompeu Fabra, Barcelona; professor titular da Faculdade de Farmácia da UFMG. Endereço eletrônico: acurcio@ medicina.ufmg.br

${ }^{7}$ Médica; doutora em Saúde Pública pela Universidade de São Paulo; professora associada da UFMG. Endereço eletrônico: cherchml@medicina.ufmg.br

Recebido em: 03/05/2010. Aprovado em: 25/02/2011. 


\section{Introdução}

A doença renal crônica tem-se apresentado, nas últimas décadas, como um problema mundial de saúde pública, pelos aumentos progressivos de sua taxa de incidência e prevalência, além de elevada morbi-mortalidade (ROMÃO-JÚNIOR, 2007). Em seu estágio mais avançado, terapias renais substitutivas (TRS - hemodiálise, diálise peritoneal ou transplante) são necessárias para manutenção da vida do paciente (ROMÃO-JÚNIOR, 2007), sendo que, no Brasil, a maioria deles se encontra na modalidade de hemodiálise (SESSO et al., 2008). As TRS, além do alto custo socioeconômico, são responsáveis por grandes repercussões sociais e psicológicas na vida do paciente submetido a elas (PIETROVSKY, 2005).

Pacientes renais crônicos em tratamento hemodialítico comumente necessitam de pelo menos três sessões semanais de terapia, com duração de aproximadamente quatro horas cada. Durante o procedimento em um serviço de diálise, enfermeiros e técnicos monitoram todo o tratamento. Para um tratamento efetivo sem intercorrências, os pacientes precisam seguir recomendações médicas quanto a restrições alimentares, diminuição de ingestão de líquidos e uso de medicamentos. Além disso, esses pacientes estão propensos a efeitos colaterais inerentes à terapia, além de problemas psicossociais, decorrentes da restrição na inserção ou adequabilidade ao mercado de trabalho e elevação do grau de dependência familiar (PIETROVSKY, 2005).

A hemodiálise é um tratamento crônico demasiadamente técnico e imprescindível à sobrevivência dos pacientes portadores de doença renal crônica terminal. Nessa perspectiva, a avaliação da satisfação desses pacientes com seu tratamento pode fornecer importantes vetores para a reorganização de serviços (PIETROVSKY, 2005; BARON-EPEL et al., 2001). Além disso, essas consequências são diferentes entre as modalidades de terapia renal substitutiva (JUERGENSEN et al., 2006).

Uma importante dimensão da avaliação da qualidade dos serviços de saúde é a satisfação de seus usuários. $\mathrm{O}$ interesse pela avaliação da satisfação com os cuidados em saúde cresceu consideravelmente nas últimas décadas, sendo possível observar diversos enfoques teóricos para sua compreensão (CROW et al., 2002; ESPERIDIĀO et al., 2006). A satisfação voltada aos usuários está relacionada a fatores médicos e não-médicos (COHEN et al., 1996) envolvendo dois aspectos: a execução de procedimentos técnicos e o intercâmbio pessoal (DONABEDIAN, 
1992). Nesse contexto, os usuários são capazes de fornecer uma avaliação dos cuidados e serviços prestados (DONABEDIAN, 1992; CROW et al., 2002).

A satisfação dos pacientes é, ao mesmo tempo, o julgamento sobre a qualidade dos cuidados em saúde que incorpora as expectativas e percepções do usuário e é resultado do cuidado vivenciado. É a reação diante do contexto, do processo e do resultado global da experiência relativa a um serviço de saúde (DONABEDIAN, 1992), podendo incluir desde alimentação, limpeza e conforto, até a qualidade técnica dos serviços (ESPERIDIÃO et al., 2006). A satisfação do paciente tornase também uma característica estrutural do serviço de saúde (DONABEDIAN, 1992). Assim, embora subjetiva, cognitivamente baseada e influenciada pelas expectativas pessoais, a perspectiva do paciente fornece informações primárias frequentemente utilizadas para avaliação da qualidade dos serviços de saúde (KLOETZEL et al., 1998; CROW et al., 2002; ESPERIDIÃO et al., 2006).

Um método comum para avaliação da satisfação é a coleta indireta de informações dos usuários a respeito da qualidade dos serviços que eles recebem, ou através do relato de suas experiências (CROW et al., 2002). Acredita-se que de forma indireta, ou através de uma solicitação repetida, os usuários sejam forçados a refletir sobre sua satisfação de forma mais assertiva (DONABEDIAN, 1992). A avaliação da satisfação do paciente em TRS incluiria, assim, informações relacionadas à comunicação e relacionamento entre equipe responsável pelo cuidado e paciente, a atmosfera da unidade de diálise e a eficiência dos suprimentos fornecidos (COHEN et al., 1996; WASSERFALLEN, 2006). Trad et al. (2002) reforçam que há clara associação entre maior acesso à equipe médica e à informação quanto à doença, proteção e cuidados e maior grau de satisfação. Conforme sugerem Argentero et al. (2008), os níveis de estresse de médicos e enfermeiros podem influenciar negativamente os níveis de satisfação do paciente com o cuidado prestado. Ainda, segundo Plantinga et al. (2004), menores níveis de satisfação estão associados a menor frequência de contato com o nefrologista.

É importante ressaltar, ainda, que embora o nível de satisfação do usuário com o tratamento esteja relacionado e impacte na qualidade de vida do pacientes em hemodiálise (PIETROVSKY, 2005; BARON-EPEL et al., 2001), trata-se de construtos distintos em relação à avaliação dos serviços.

Qualidade de vida, conforme definida pela Organização Mundial da Saúde (OMS), é a percepção individual de uma posição de vida deste indivíduo em um 
contexto cultural e de valores, permeado dos objetivos pessoais, expectativas, padrōes e conceitos nos quais estes indivíduos vivem. É um conceito dinâmico, profundamente afetado pela saúde física, estado psicológico, nível de independência, relações sociais, crenças pessoais/espiritualidade/religião e características ambientais - por exemplo, seguridade, recursos financeiros e liberdade (WHO, 1997). A qualidade de vida poderia então ser entendida como a percepção individual de bem-estar, sendo transmitida pelo nível de satisfação ou insatisfação em relação a cada um dos domínios da vida e a importância que se dá a eles, sendo uma avaliação que a própria pessoa faz a respeito de sua saúde e de sua vida (SAUPE; BROCA, 2004; RUDNICKI, 2007).

A qualidade de vida é algo, portanto, que se confunde com o próprio conceito de saúde definido pela OMS (WHO, 1997). Observa-se que, embora a satisfação do usuário com o tratamento seja permeada de muitos domínios de vida que também contribuam para uma avaliação da qualidade de vida, a avaliação da qualidade de vida é um indicador em saúde estruturalmente mais complexo e genérico.

Atentando para essas particularidades, a avaliação da satisfação dos pacientes em hemodiálise é um importante domínio, pois pode estar associada à adesão ao tratamento(KUTNERetal.,2005;ESPERIDIÃOetal.,2006; WASSERFALLEN et al., 2006) e afetar os resultados em saúde (WASSERFALLEN et al., 2006). É, portanto, parte integrante das redes de monitoramento do cuidado, na tentativa de se produzir uma medida útil que auxilie a tomada de decisões no âmbito dos serviços (ESPERIDIÃO et al., 2006). Há, no entanto, relativamente poucos estudos que avaliam a satisfação do paciente em hemodiálise no Brasil.

O objetivo deste estudo foi avaliar os fatores socioeconômicos, demográficos, clínicos e estruturais dos serviços de diálise associados à satisfação dos pacientes portadores de doença renal crônica terminal em hemodiálise com o cuidado recebido nos serviços de diálise, no Brasil, no ano de 2007.

\section{Métodos}

\section{Participantes}

Os dados utilizados no trabalho são provenientes de pesquisa realizada pelo Projeto TRS - Avaliação Econômico-Epidemiológica das modalidades de Terapia Renal Substitutiva no Brasil, conduzido pelo Grupo de Pesquisa em Economia da Saúde da Universidade Federal de Minas Gerais (GPES/UFMG), entre os 
meses de janeiro e maio de 2007. Foi selecionada uma amostra representativa

de pacientes em tratamento renal substitutivo, atendidos em serviços de diálise e centros transplantadores. Os pacientes responderam (no local de tratamento) a questionário estruturado aplicado por entrevistadores selecionados e previamente treinados. O questionário aplicado contemplava informações socioeconômicas, demográficas e de qualidade de vida. Aqueles que se encontravam na modalidade de hemodiálise, no momento da entrevista, responderam também a um questionário específico de satisfação elaborado e utilizado por Roderick et al. (2005), foco da presente investigação. Foram incluídos pacientes maiores de 18 anos, portadores de doença renal crônica terminal, mantidos em tratamento de diálise há pelo menos três meses ou transplantados há pelo menos seis meses. Pacientes retransplantados ou com história de transplantes múltiplos foram excluídos. A amostragem foi feita por conglomerado em dois estágios: serviços de diálise e pacientes. Todos os participantes assinaram termo de consentimento livre e esclarecido.

\section{Entrevista}

O questionário de satisfação utilizado foi desenvolvido por Roderick et al. (2005). É composto por 22 questôes fechadas, que envolvem a relação dos pacientes com a equipe de diálise, o impacto da diálise em suas atividades diárias, além de informações quanto ao ambiente de diálise e atendimento do serviço, tendo todas como opçôes de resposta "sim" e "não".

\section{Análise estatística}

Foi realizada uma análise descritiva das variáveis socioeconômicas, demográficas e estruturais dos serviços de diálise e dos 22 itens do questionário de satisfação. Os modelos de Teoria de Resposta ao Item (TRI) ou Teoria do Traço Latente foram aplicados aos itens desse questionário.

Essa técnica utiliza modelos matemáticos para a construção de indicadores baseados em um conjunto de variáveis observadas (cada um dos 22 itens do questionário) e variáveis hipotéticas, ditos traços latentes (no caso, a satisfação). Acredita-se que todo desempenho comportamental traz um conjunto subjacente de traços latentes, que serão indiretamente observados (PASQUALI et al., 2003). Logo, assumiu-se que a satisfação do usuário em hemodiálise pode ser quantificada como uma variável latente. As opções de respostas aos itens 
do questionário de satisfação assumiram valores 0 e 1, sendo " 0 " uma resposta negativa e "1" positiva, de acordo com cada item.

Inicialmente, a avaliação da correlação bisserial foi realizada a fim de verificar a correlação entre cada um dos itens do questionário com o escore bruto produzido pelo conjunto das variáveis. A partir dessa análise, foi possível selecionar as questôes que apresentavam consistência interna e se associavam significativamente ao escore de satisfação produzido. Sugere-se que itens com valores de correlação bisserial inferiores a 0,3 devam ser excluídos de qualquer análise posterior (SOARES, 2005).

Procedeu-se, então, à investigação da unidimensionalidade da escala, admitindo a existência de um único traço latente responsável pelo desempenho no conjunto de itens do questionário (PASQUALI et al., 2003; SOARES, 2005). Para cada um dos itens selecionados, o modelo logístico de um parâmetro (Rasch) e o de dois parâmetros foram ajustados. Os parâmetros utilizados por esses modelos incluem: o guessing parameter, que expressa a probabilidade de um indivíduo com habilidade muito baixa responder corretamente ao item pelo acaso; o parâmetro de discriminação que quantifica o quanto determinado item distingue indivíduos com baixo e alto padrão de escala latente; e o parâmetro de dificuldade, que expressa o nível de dificuldade do item. Na prática, o parâmetro de dificuldade vai de $-3 \mathrm{a}+3$, indicando o ponto na escala de aptidão com $50 \%$ de probabilidade de ser uma resposta correta. Assim, quanto maior o valor do parâmetro, maior deve ser a aptidão ou expressão comportamental do indivíduo para que ele tenha "chance", de $50 \%$, de responder o item como esperado. Para o parâmetro de discriminação, a métrica vai de 0 a 3 , onde 0 significa nenhuma discriminação e 3 uma discriminação perfeita. (PASQUALI et al., 2003; RIZOPOULOS, 2006).

O modelo de Rasch assume que, provavelmente, nenhum indivíduo responda de maneira esperada ao acaso (guessing parameter igual a 0) e que o parâmetro de discriminação é igual a 1 . O modelo de dois parâmetros (parâmetro de dificuldade e de discriminação) também assume que responder conforme esperado ao acaso é improvável e permite diferentes valores tanto para o parâmetro de dificuldade quanto para o parâmetro da discriminação. (PASQUALI et al., 2003; RIZOPOULOS, 2006).

Verificou-se então, dentre os modelos, qual apresentava melhor ajuste, e valendo-se do modelo escolhido, os escores de satisfação puderam ser gerados 
(RIZOPOULOS, 2006), com intervalo de variação entre 0 e 1 , sendo "0" o mais

baixo nível de satisfação e "1" o mais alto nível. A partir dos escores obtidos, foi utilizada regressão linear univariada para verificar quais fatores socioeconômicos, demográficos e clínicos dos pacientes, além de características dos serviços de diálise, estariam associados ao nível de satisfação de pacientes em hemodiálise ( $\mathrm{p} \leq 0,05)$. Aqueles estatisticamente significantes foram testados através de regressão linear múltipla $(\mathrm{p} \leq 0,05)$ para composição do modelo final. A qualidade do ajuste foi verificada pela análise dos resíduos. O programa estatístico utilizado foi o R 2.10.1.

Esse projeto foi aprovado pelo Comitê de Ética em Pesquisa da Universidade Federal de Minas Gerais (Parecer ETIC 397/04).

\section{Resultados}

\section{Caracterização da amostra e da satisfação do paciente em hemodiálise}

Foram entrevistados 3.036 pacientes em TRS, sendo que 1.621 se encontravam em hemodiálise no momento da entrevista e responderam ao questionário de satisfação proposto por Roderick et al. (2005). A proporção de resposta a essa parte da entrevista foi de $100 \%$.

A média de idade dos pacientes entrevistados foi de 48,9 $\pm 14,49$ anos. A renda apresentou média de $\mathrm{R} \$ 2312,00 \pm 3339,28$, e mediana de $\mathrm{R} \$ 623,00$ (o correspondente a dois salários mínimos da época). O tempo de tratamento dialítico médio foi de aproximadamente 4,5 anos e o tempo gasto de sua residência até a unidade de diálise foi de $45 \pm 39$ minutos. Outras características socioeconômicas, demográficas e clínicas dos pacientes, além de informações dos serviços de diálise, são apresentadas na tabela 1. A maioria dos pacientes respondeu positivamente em relação à satisfação relacionada aos itens do questionário. A tabela 2 apresenta os itens do questionário, assim como as proporções das opções de resposta para cada um desses itens.

Observam-se níveis de satisfação acima de $80 \%$ de satisfação para quase todos os itens. Percentuais mais baixos de satisfação foram encontrados para acesso a medicamentos de uso contínuo $(75,6 \%)$ e de medicamentos no serviço público $(49,8 \%)$. Quanto à repercussão da diálise na própria vida social e vida social do parceiro, a maioria dos pacientes respondeu que a hemodiálise atrapalha, lembrando que, neste caso, a resposta positiva repercurte em menor satisfação em relação a este item. 


\section{Teoria de Resposta ao Item}

A avaliação da correlação bisserial revelou três itens (P 19, P 20 e P 22 - tabela 2) com valor inferior a 0,3 (respectivamente, 0,$156 ; 0,253$ e 0,23 ) e, portanto, estes itens foram excluídos de análises posteriores, acreditando-se que por meio deste ponto de corte, o fato de fazer diálise sempre com o mesmo grupo (P 19), o gostar de fazer diálise com outros pacientes (P 20) e oferecimento de lanche pela clínica de diálise (P 22) não apresentam consistência interna suficiente para estarem associados ao escore de satisfação que será produzido.

$\mathrm{O}$ teste de unidimensionalidade apresentou valor-p igual a 0,05 , o que confirma a existência de um fator geral de mensuração para a satisfação com a aplicação desse questionário.

O modelo de dois parâmetros apresentou melhor ajuste que o de Rasch, e então foi utilizado para gerar os escores de satisfação, conforme apresentado na tabela 3. A investigação da qualidade de cada um dos itens avaliados no modelo de dois parâmetros mostrou que os valores do parâmetro de discriminação variaram entre 0,63 a 3,232, sendo o valor menor (baixa discriminação) referente ao item privacidade ao fazer diálise e o maior valor referente a boa relação com enfermeiro (alto poder discriminativo). Quanto ao parâmetro de dificuldade, os valores variaram entre $-2,22$ a 0,14 . O menor valor foi apresentado pelo item relacionado à privacidade ao fazer diálise, o que significa que este item é considerado fácil de ser respondido, enquanto o maior grau de dificuldade foi encontrado para o item "diálise atrapalha vida social do paciente". No geral, nenhum item apresentou grau de dificuldade elevado (próximo de 3).

Após a estimação desses parâmetros, o escore de satisfação foi desenvolvido e os fatores individuais e dos serviços de diálise associados ao nível de satisfação de pacientes em hemodiálise puderam ser avaliados por meio da regressão linear univariada e estão apresentados na tabela 4.

A regressão linear múltipla revelou quatro fatores associados a menores níveis de satisfação (escores mais baixos de satisfação): menor idade, ter relatado depressão, maior tempo de tratamento dialítico e maior tempo de percurso entre residência e serviço de diálise (tabela 5). A análise dos resíduos mostrou que o modelo foi bem ajustado, uma vez que eles apresentaram média zero e comportamento homocedástico. 
A análise da satisfação do usuário nessa investigação identificou que menores níveis de satisfação estão associados a menor idade, depressão, maior tempo de tratamento dialítico e maior tempo de percurso entre a residência e o serviço de diálise.

Acerca da relação entre satisfação e idade, conforme discorrem Crow et al. (2002), de 58 estudos que investigaram essa relação, 41 (70,7\%) encontraram que pacientes mais velhos são significativamente mais satisfeitos, 13 (22,4\%) não encontraram relação e o restante identificou maior satisfação para pacientes mais jovens. Pessoas de maior idade poderiam ser menos exigentes ou mais complacentes com o cuidado providenciado, ou ainda, devido a experiências anteriores teriam padrôes de exigência mais baixos (CROW et al., 2002).

Quanto à depressão, pacientes com alteração do estresse psicológico tendem a apresentar uma percepção adulterada dos cuidados em saúde o que também incide em uma visão depreciada das relaçôes interpessoais (WILSON et al., 1995). Além disso, como agravante, pessoas com depressão tendem a negligenciar sua adesão às medidas de cuidado estabelecidas (KIMMEL et al., 1998) tornandose necessário que os profissionais envolvidos na atenção revistam sua prática de maior tolerância e sensibilidade.

Além disso, observou-se que quanto maior o tempo de tratamento dialítico, menor o nível de satisfação. Os dados apresentados vão na direção dos resultados do estudo realizado por Bakewell et al. (2002), que verificou alteração das várias dimensões da qualidade de vida, dentre os quais a satisfação do paciente com o aumento do número de meses de tratamento de pacientes em diálise peritoneal. Isto pode ser resultado do desenvolvimento de um sentimento de frustração em relação à doença, à medida que os anos passam, o que influenciaria negativamente os níveis de satisfação. No entanto, diversos estudos não encontram diferenças significativas para diferentes tempos de tratamento. Sugere-se que o estado de saúde pode ser um importante mediador dessa relação (CROW et al., 2002).

E ainda, a satisfação revelou-se associada ao tempo de percurso entre moradia e serviço de diálise. Segundo Willis et al. (1998), este é um importante aspecto a ser avaliado para pacientes em tratamento dialítico, uma vez que já estão sujeitos a um tratamento crônico, que absorve grande parte de seu tempo. Assim, um maior tempo gasto nesse trajeto diminuiria ainda mais o tempo disponível 
para outras atividades, podendo inclusive incidir sobre horas de sono. Somamse a esse fato os gastos com o transporte que podem aumentar dependendo da localização do serviço, ou a disponibilidade/indisponibilidade de transporte gratuito. Sugere-se, então, que este deva ser um critério importante na alocação dos pacientes entre os diversos serviços de diálise, de forma que os pacientes sejam atendidos em unidades mais próximas de sua residência. No sistema de saúde inglês, National Health Service (NHS), é estabelecido que no máximo 30 minutos sejam despendidos no trajeto entre a residência do paciente e a clínica de diálise. Este tempo só poderia ser ultrapassado em horários de tráfico mais intenso (THE RENAL ASSOCIATION, 2009).

Esta investigação possui limitações como a utilização de questionário estruturado que possivelmente não envolve todos os componentes da avaliação da satisfação e a aplicação do questionário no momento da terapia, no serviço de diálise, o que introduz a possibilidade de inibição do respondente, mesmo que o entrevistador atentasse o paciente para o anonimato de sua entrevista. Em relação ainda às informações obtidas e ao método de análise, a utilização da Teoria de Resposta ao Item para gerar os escores de satisfação mostrou-se adequada para a avaliação pretendida, o que não exclui a possibilidade e a necessidade de que novos itens com maior nível de complexidade sejam elaborados e culminem em instrumentos mais discriminativos na avaliação deste importante indicador em saúde.

Refletindo acerca desses achados, observa-se que o processo saúde/doença é permeado de elementos socioeconômicos e culturais, podendo ser vivenciado diferentemente pelos vários atores que dele participam. Da mesma forma, a avaliação dos serviços de saúde estaria influenciada por determinados valores, ideologias, crenças, posições de classe, dentre outros. A unidade de análise torna-se, portanto, a totalidade que o serviço representa, sendo necessário considerar as conexões existentes entre os vários elementos que a determinam (DESLANDES, 1997). A teorização no campo da avaliação dos serviços de saúde busca converter os conceitos em estratégias e critérios que subsidiarão tomadas de decisão (ESPERIDIÃO, 2006). Assim, a avaliação de serviço pela satisfação do usuário reveste-se dessa complexidade, propondo que o processo avaliativo de suas dimensões não seja medido com presteza, ou suficiência absoluta, mas que possa viabilizar informações importantes para melhoria da qualidade da atenção prestada. 
Quanto ao contexto em que este indicador é avaliado, há também importantes limitações a serem consideradas. Há que se compreender que estes pacientes têm a atenção garantida por um sistema público de saúde que conta neste setor com prestadores em maioria privados, somado à sofisticação tecnológica de empresas também privadas estrangeiras que fornecem tanto as máquinas de hemodiálise quanto a maioria dos insumos utilizados (SBN, 2008), o que repercute em interesses e tensões específicos. Qualificar a escuta das necessidades destes pacientes de forma a sistematizar propostas de avaliação constante da satisfação destes usuários e, consequentemente, criar oportunidades de melhorar a atenção prestada que considerem e respeitem a integralidade do cuidado que o sistema de saúde preconiza é uma tarefa árdua e delicada.

Evidenciou-se neste estudo que a avaliação da satisfação do usuário não envolve apenas fatores individuais, mas está também relacionada a atributos dos serviços de saúde, confirmando sua natureza multidimensional. Sem esgotar as possibilidades de avaliação de serviços de saúde, os resultados encontrados para a satisfação do usuário são capazes de apontar importantes pontos de atuação tanto para iniciativas locais dos profissionais e prestadores de serviço quanto para os gestores. Iniciativas que possam garantir suporte para o enfretamento das peculiaridades desse tratamento e, assim, providenciem aumento da qualidade da atenção prestada a esses pacientes. Atuar dessa forma exige uma nova perspectiva dos profissionais envolvidos no cuidado, que vai além do aprimoramento técnico, conduzindo à necessidade de aperfeiçoamento do processo assistencial em busca da integralidade e humanização. Outras investigaçôes que aprofundem essa discussão sob a ótica dos métodos qualitativos devem ser desenvolvidas.

\section{Referências}

ARGENTERO, P. et al. Staff burnout and patient satisfaction with the quality of dialysis care. Am J Kidney Dis, v. 51, n. 1, p. 80-92, 2008.

BAKEWEEL, A.B.; HIGGINS, R.M., EDMUNDS, M.E. Quality of life in peritoneal dialysis patients: declive over time and association with clinical outcomes. Kid Int, v. 61, p.239-48, 2002.

BARON-EPEL, O.; DUSHENAT, M.; FRIEDMAN, N. Evaluation of the consumer model: relationship between patients' expectations, perceptions and satisfaction with care. Int J Qual Heatlh Care, v. 13, n. 4, p. 317-23, 2001. 
COHEN, G.; FORBES, J.; GARRAWAY, M. Can different patient satisfaction survey methods yield consistent results? Comparison of three surveys. $\mathrm{Br}$ Med J, v. 313, n. 7061, p. 841-4, 1996.

CROW, R. et al. The measurement of satisfaction with healthcare: implications for practice from a sistematic review of the literature. Health Technol Assesst., v. 6, n. 32, p. 1-244, 2002. DONABEDIAN, A. Quality assurance in health care: consumers' role. Qual Health Care, v. 1, p. 247-51, 1992.

ESPERIDIÃO, M. A.; TRAD, L.A.B. Avaliação de satisfação de usuários: considerações teórico-conceituais. Cad. Saúde Pública, Rio de Janeiro, v. 22, n. 6, p. 1267-76, 2006.

JUERGENSEN, E. et al. Clin J Am Soc Nephrol., v. 1, p. 1191-6, 2006.

KIMMEL, P. L. et al. Psychosocial factors, behaviour compliance and survival in urban hemodialysis patients. Kid Int., v. 54, p. 245-54, 1998.

KLOETZEL, K. et al. Controle de qualidade em atenção primária à saúde. I - A satisfação do usuário. Cad. Saúde Pública, Rio de Janeiro, v. 14, n. 3, p. 623-8, 2006.

KUTNER, N. G.; ZHANG, R.; BROGAN, D. Race, gender, and incident dialysis patient's reported health status and quality of life. J Am Soc Nephrol., v. 16, p. 1440-8, 2005.

PASQUALI, L.; PRIMI, R. Fundamentos da Teoria da Resposta ao Item - TRI. Avaliação Psicológica, v. 2, n. 2, p. 99-110, 2003.

PIETROVSKI, V. O espaço da hemodiálise na ótica dos usuários com insuficiência renal crônica. 2005. 76p. Dissertação (Mestrado em Enfermagem) - Escola de Enfermagem, Universidade Federal do Rio Grande do Sul, Porto Alegre, 2005.

PLANTINGA, L.C. et al. Frequency of patient-physician contact and patient outcomes in hemodialysis care. J Am Soc Nephrol., v. 15, p. 210-8, 2004.

RIZOUPOULOS, D. LTM: an R Package for Latent Variable Modeling and Item Response Theory Analyses. J of Stat Softw., v. 17, n. 5, p. 1-25, 2006.

RODERICK, P. et al. An evaluation of the costs, effectiveness and quality of renal replacement therapy provision in renal satellite units in England and Wales. Health Technol Assess., v. 9, n. 24, p. 1-178, 2005.

ROMÃO-JÚNIOR, J. E. A doença renal crônica: do diagnóstico ao tratamento. Prática hospitalar, v. 52, p. 183-187, jul-ago 2007.

RUDNICKI, T. Preditores de qualidade de vida em pacientes renais crônicos. Estudos de Psicologia, Campinas, v. 24, n. 3, p. 343-51, 2007.

SAUPE, R.; BROCA G. S. Indicadores de qualidade de vida como tendência atual de cuidado a pessoas em hemodiálise. Texto e Contexto Enfermagem, v. 13, n. 001, p. 100-6, 2004. 
SESSO, R. et al. Brazilian Dialysis Census Report, 2008. J Bras Nefrol, São Paulo, v. 30, n. 4, p. 233-238, 2008.

SOARES, T.M. Utilização da Teoria da Resposta ao Item na produção de indicadores sócioeconômicos. Pesquisa Operacional, v. 25, n. 1, p. 83-112, 2005.

THE RENAL ASSOCIATION. Haemodialysis Final Draft. Current verion. Dec 2009.

Disponível em: <http://www.renal.org/Clinical/GuidelinesSection/Haemodialysis. aspx\#downloads>. Acesso em: 09 abr. 2010.

TRAD, L.A.B. et al. Estudo etnográfico da satisfação do usuário do Programa de Saúde da Família (PSF) na Bahia. Cien Saúde Col., v. 7, n. 3, p. 581-9, 2002.

WASSERFALLEN, J. et al. Satisfaction of patients on chronic haemodialysis and peritoneal dialysis. Swiss Med Wkly., v. 136, p. 210-7, 2006.

WILLIS, C. E. et al. Location for renal services: patient satisfaction surveys. Ulster Med J, v. 67 , n. 2, p. 110-4, 1998.

WILSON, P. M. J. et al. Examination of the effects of emotional disturbance and its detection on general practice patients' satisfaction with the consultation. BrJ Gen Pract., v. 45, p. 304-9, 1995.

WORLD HEALTH ORGANIZATION. WHOQOL Measuring Quality of Life: the World Health Organization Quality of life Instruments. 1997. Disponível: http://www.who.int/ mental_health/media/68.pdf Acesso em: 26 dez 2010. 
Tabela 1. Características da população de pacientes em hemodiálise, Brasill, 2007

\begin{tabular}{|c|c|c|}
\hline População & $\mathbf{N}$ & $\%$ \\
\hline Total & 1621 & 100,00 \\
\hline \multicolumn{3}{|c|}{ Indivíduos } \\
\hline \multicolumn{3}{|l|}{ Sociodemográficas e econômicas } \\
\hline \multicolumn{3}{|l|}{ Sexo } \\
\hline Masculino & 914 & 56,38 \\
\hline Feminino & 707 & 43,62 \\
\hline \multicolumn{3}{|l|}{ Cor da pele } \\
\hline Não branco & 981 & 60,52 \\
\hline Branco & 622 & 38,37 \\
\hline Não sabe & 18 & 1,10 \\
\hline \multicolumn{3}{|l|}{ Escolaridade } \\
\hline$\leq 2^{\circ}$ grau & 1457 & 89,88 \\
\hline$>2^{\circ}$ grau & 160 & 9,87 \\
\hline Não sabe & 4 & 0,25 \\
\hline \multicolumn{3}{|l|}{ Estado civil } \\
\hline Solteiro(a) & 385 & 23,75 \\
\hline Casado(a)/Vivendo como casado & 921 & 56,82 \\
\hline Separado(a) & 99 & 6,11 \\
\hline Divorciado(a) & 83 & 5,12 \\
\hline Viúvo(a) & 130 & 8,02 \\
\hline Outro & 3 & 0,19 \\
\hline \multicolumn{3}{|l|}{ Filhos } \\
\hline Possui & 1275 & 78,66 \\
\hline Não possui & 345 & 21,28 \\
\hline Não sabe & 1 & 0,06 \\
\hline \multicolumn{3}{|l|}{ Classe social } \\
\hline A e B & 396 & 24,43 \\
\hline $\mathrm{C}$ & 671 & 41,39 \\
\hline $\mathrm{D}$ e $\mathrm{E}$ & 550 & 33,93 \\
\hline Não sabe & 4 & 0,20 \\
\hline \multicolumn{3}{|l|}{ Plano de saúde } \\
\hline Possui & 473 & 29,18 \\
\hline Não possui & 1145 & 70,64 \\
\hline Não sabe & 3 & 0,19 \\
\hline
\end{tabular}




\begin{tabular}{|c|c|c|}
\hline \multicolumn{3}{|l|}{ Clínica } \\
\hline \multicolumn{3}{|l|}{ Depressão } \\
\hline Não & 1322 & 81,55 \\
\hline Sim & 294 & 18,14 \\
\hline Não sabe & 4 & 0,30 \\
\hline \multicolumn{3}{|l|}{ Hipertensão arterial } \\
\hline Não & 263 & 16,22 \\
\hline $\operatorname{Sim}$ & 1356 & 83,65 \\
\hline Não sabe & 2 & 0,10 \\
\hline \multicolumn{3}{|l|}{ Diabetes mellitus } \\
\hline Não & 1250 & 77,11 \\
\hline $\operatorname{Sim}$ & 367 & 22,64 \\
\hline Não sabe & 4 & 0,20 \\
\hline \multicolumn{3}{|l|}{ Modalidade de início } \\
\hline Hemodiálise & 1466 & 90,4 \\
\hline Diálise peritoneal & 152 & 9,4 \\
\hline Transplante & 2 & 0,1 \\
\hline Não sabe & 1 & 0,1 \\
\hline \multicolumn{3}{|c|}{ Mudança de modalidade } \\
\hline Sim & 91 & 5,61 \\
\hline Não & 1526 & 94,14 \\
\hline Não sabe & 4 & 0,25 \\
\hline \multicolumn{3}{|l|}{ Serviços de diálise } \\
\hline \multicolumn{3}{|c|}{ Características da unidade de diálise } \\
\hline \multicolumn{3}{|c|}{ Tipo de unidade } \\
\hline Ambulatorial & 998 & 61,57 \\
\hline Policlínica & 20 & 1,23 \\
\hline Hospitalar & 603 & 37,20 \\
\hline \multicolumn{3}{|l|}{ Esfera-administrativa } \\
\hline Privada & 1468 & 90,56 \\
\hline Pública & 153 & 9,44 \\
\hline
\end{tabular}

Fonte: Projeto TRS, 2007 
Tabela 2. Satisfação de pacientes em hemodiálise, Brasil, 2007

\begin{tabular}{|c|c|c|c|}
\hline \multicolumn{2}{|r|}{ Itens* } & $\mathbf{N}$ & $\%(\operatorname{sim})$ \\
\hline P1 & Boa relação com médico & 1539 & 94,9 \\
\hline P2 & Boa relação com Enfermeiro & 1567 & 96,7 \\
\hline P3 & Sente Seguro & 1522 & 93,9 \\
\hline P4 & Confio na capacidade dos enfermeiros de cuidar de mim & 1516 & 93,5 \\
\hline P5 & Tempo suficiente para discutir com enfermeiros & 1386 & 85,5 \\
\hline P6 & Fácil marcar consulta com meu médico & 1440 & 88,8 \\
\hline P7 & Tem oportunidade de discutir problemas com o médico & 1398 & 86,2 \\
\hline P8 & $\begin{array}{l}\text { Recebe informação sobre o quer saber sobre sua } \\
\text { doença renal e tratamento }\end{array}$ & 1475 & 91,0 \\
\hline P9 & Fácil acesso a medicamentos uso contínuo & 1225 & 75,6 \\
\hline P10 & Fácil acesso a medicamentos no serviço público & 807 & 49,8 \\
\hline P11 & Fácil acesso a assistente social & 1341 & 82,7 \\
\hline P12 & Fácil acesso a nutricionista & 1308 & 80,7 \\
\hline P13 & Diálise atrapalha vida social do paciente & 834 & 51,4 \\
\hline P14 & Diálise atrapalha vida social do parceiro & 581 & 35,8 \\
\hline P15 & Clínica de diálise tem ambiente agradável & 1540 & 95,0 \\
\hline P16 & Clínica de diálise tem ambiente calmo & 1477 & 91,1 \\
\hline P17 & Clínica de diálise tem espaço bem distribuído & 1478 & 91,2 \\
\hline P18 & Possui privacidade ao fazer diálise & 348 & 21,5 \\
\hline P19 & Faz diálise com o mesmo grupo de pacientes todos os dias & 1567 & 96,7 \\
\hline P20 & $\begin{array}{l}\text { Gosta de encontrar outros pacientes da sua sessão } \\
\text { quando vem para diálise }\end{array}$ & 1558 & 96,1 \\
\hline P21 & Facilidade de atendimento na clínica quando se sente mal & 1435 & 88,5 \\
\hline P22 & Clínica oferece lanche & 1385 & 85,4 \\
\hline
\end{tabular}

Fonte: Projeto TRS, 2007

* Roderick et al, 2005. 

o método da Teoria de Resposta ao Item para questionário de satisfação de pacientes em hemodiálise*, Brasil, 2007

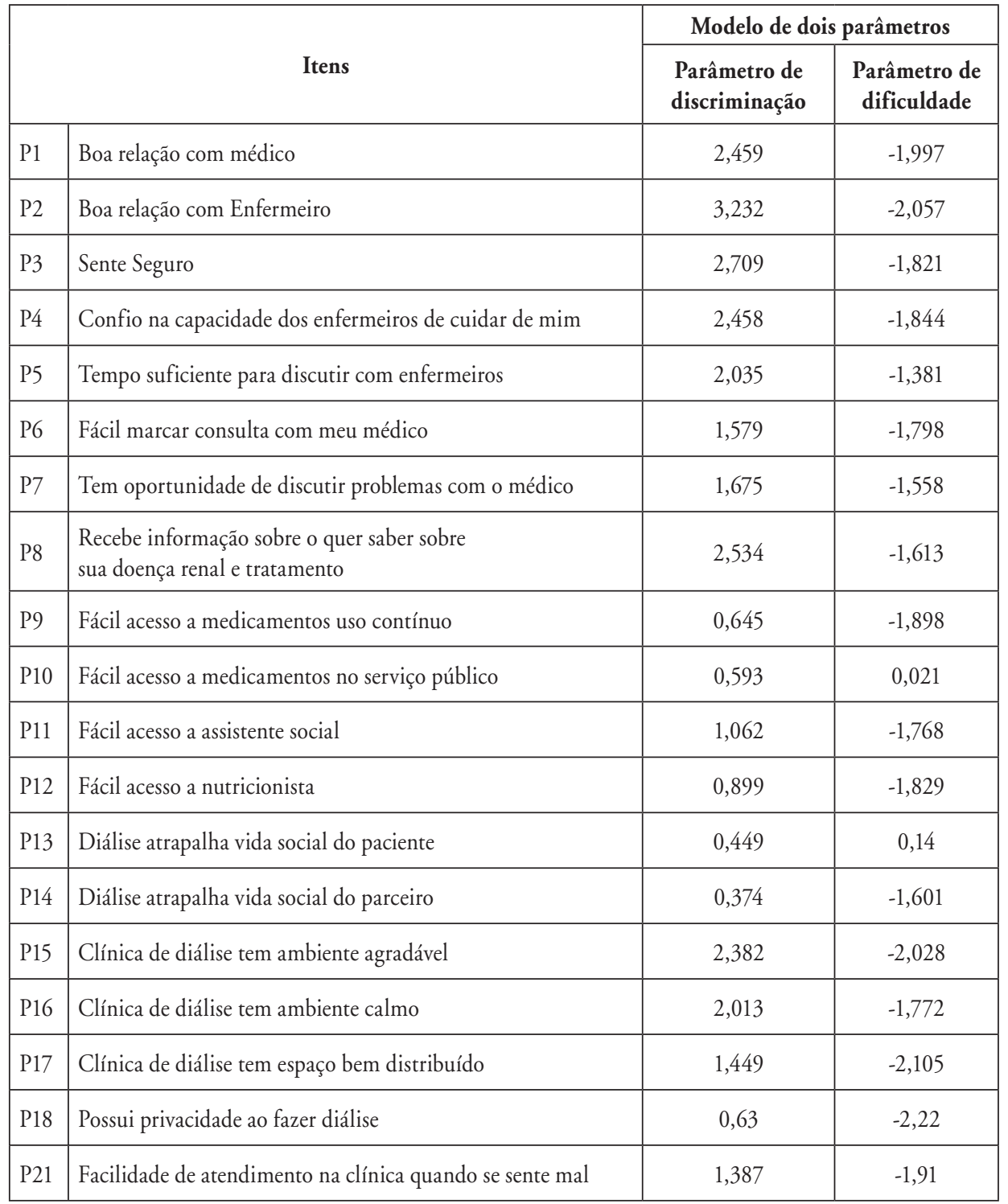

Fonte: Projeto TRS, 2007

* Roderick et al, 2005. 
Tabela 4. Regressão linear univariada da satisfação de pacientes em hemodiálise com outros fatores, Brasil, 2007

\begin{tabular}{|c|c|}
\hline Características & valor-p \\
\hline \multicolumn{2}{|l|}{ Indivíduos } \\
\hline \multicolumn{2}{|l|}{ Socioeconômicas e demográficas } \\
\hline Sexo & 0,441 \\
\hline Idade & * \\
\hline Cor da pele & $*$ \\
\hline Escolaridade & 0,849 \\
\hline Estado civil & * \\
\hline Filhos & 0,908 \\
\hline Classe social & 0,122 \\
\hline Renda & 0,221 \\
\hline Plano de saúde & 0,380 \\
\hline Tempo de percurso entre residência e serviço de diálise & * \\
\hline \multicolumn{2}{|l|}{ Clínica } \\
\hline Depressão & * \\
\hline Hipertensão arterial & 0,662 \\
\hline Diabetes mellitus & 0,281 \\
\hline Modalidade de início & 0,307 \\
\hline Mudança de modalidade & 0,600 \\
\hline Tempo de tratamento dialítico & $*$ \\
\hline \multicolumn{2}{|l|}{ Serviços de diálise } \\
\hline \multicolumn{2}{|l|}{ Características da unidade de diálise } \\
\hline Tipo de unidade & 0,276 \\
\hline Esfera-administrativa & 0,232 \\
\hline
\end{tabular}

Fonte: Projeto TRS, 2007

${ }^{*} \mathrm{p}<0,05$ 
Tabela 5. Regressão linear múltipla da satisfação de pacientes em hemodiálise com outros fatores, Brasil, 2007

\begin{tabular}{|l|c|c|}
\hline & Coeficiente $(\beta)$ & valor-p \\
\hline Idade & 0,151 & 0,000 \\
\hline Depressão & 0,590 & 0,003 \\
\hline Tempo de tratamento dialítico & $-0,072$ & 0,018 \\
\hline Tempo de percurso entre residência e serviço de diálise & $-0,078$ & 0,000 \\
\hline
\end{tabular}

Fonte: Projeto TRS, 2007 


\section{Assessment of the satisfaction of patients in chronic hemodialysis with the treatment at dialysis services in Brazil}

This study aimed to evaluate the satisfaction of patients on chronic hemodialysis with the service provided in dialysis units. This cross-sectional study was developed in a nationally representative sample of patients with terminal renal disease $(\mathrm{N}=3,036)$ treated at dialysis units and transplant centers in Brazil. Patients in hemodialysis (HD) answered a satisfaction questionnaire designed for this modality, whose issues comprised satisfaction with staff, environment and health care management, and impact of dialysis in daily activities. The item response theory was applied in the analysis of the 22 questions of the satisfaction questionnaire to obtain a score of satisfaction. Linear regression was used to evaluate the association between socio-economic, demographic, clinical and structural characteristics of dialysis services and satisfaction scores. Of the patients interviewed, 1,621 were in HD at the time of interview. All of them answered the questionnaire of satisfaction; most were male, non-white skin color, $\leq 11$ years of education, and did not report depression. The mean age was 49 years. Data analysis showed that younger patients younger, reporting depression, with longer time of dialysis and who spent more time from home to the dialysis services were associated with lower satisfaction. It is suggested that, to assess user's satisfaction, it is important to consider, beyond individual factors, the attributes related to health services which reinforce its multidimensional nature.

> Key words: user's satisfaction; kidney failure; item response theory; health evaluation. 